\title{
ORIGINAL ARTICLE Short telomere length is associated with impaired cognitive performance in European ancestry cohorts
}

S Hägg ${ }^{1}$, Y Zhan ${ }^{1}$, R Karlsson ${ }^{1}$, L Gerritsen ${ }^{1}$, A Ploner ${ }^{1}$, SJ van der Lee ${ }^{2}$, L Broer $^{3}$, J Deelen ${ }^{4,5}$, RE Marioni $^{6,7,8}$, A Wong $^{9}$, A Lundquist $^{10}$, $\mathrm{G} \mathrm{Zhu}^{11}$, NK Hansell ${ }^{8,11}$, E Sillanpää ${ }^{12}$, IO Fedko ${ }^{13}$, NA Amin ${ }^{2}$, M Beekman ${ }^{4}$, AJM de Craen ${ }^{14, w^{4}}$, S Degerman ${ }^{15}$, SE Harris $^{6,7}, \mathrm{~K}^{-J} \mathrm{Kan}^{13}{ }^{13}$ CM Martin-Ruiz ${ }^{16}$, GW Montgomery ${ }^{11}$, NeuroCHARGE Cognitive Working Group, AN Adolfsson ${ }^{17}$, CA Reynolds ${ }^{18}$, NJ Samani ${ }^{19,20}$, HED Suchiman ${ }^{4}$, A Viljanen ${ }^{12}$, T von Zglinicki ${ }^{21}$, MJ Wright ${ }^{8,22}$, J-J Hottenga ${ }^{13}$, DI Boomsma ${ }^{13}$, T Rantanen ${ }^{12}$, JA Kaprio ${ }^{23,24,25}$, DR Nyholt ${ }^{11,26}$, NG Martin ${ }^{11}$, L Nyberg ${ }^{27,28,29}$, R Adolfsson ${ }^{17}$, D Kuh ${ }^{9}$, JM Starr ${ }^{7,30}$, IJ Deary ${ }^{7,31}$, PE Slagboom ${ }^{4}$, CM van Duijn ${ }^{2}$, V Codd ${ }^{19,20}$, NL Pedersen ${ }^{1}$ for the ENGAGE Consortium

The association between telomere length (TL) dynamics on cognitive performance over the life-course is not well understood. This study meta-analyses observational and causal associations between TL and six cognitive traits, with stratifications on APOE genotype, in a Mendelian Randomization (MR) framework. Twelve European cohorts $(N=17052$; mean age $=59.2 \pm 8.8$ years) provided results for associations between qPCR-measured TL (T/S-ratio scale) and general cognitive function, mini-mental state exam (MMSE), processing speed by digit symbol substitution test (DSST), visuospatial functioning, memory and executive functioning (STROOP). In addition, a genetic risk score (GRS) for TL including seven known genetic variants for TL was calculated, and used in associations with cognitive traits as outcomes in all cohorts. Observational analyses showed that longer telomeres were associated with better scores on DSST ( $\beta=0.051$ per s.d.-increase of TL; $95 \%$ confidence interval $(C \mathrm{l}): 0.024,0.077 ; P=0.0002)$, and MMSE $(\beta=0.025 ; 95 \% \mathrm{Cl}: 0.002,0.047 ; P=0.03)$, and faster STROOP $(\beta=-0.053 ; 95 \% \mathrm{Cl}:-0.087,-0.018 ; P=0.003)$. Effects for DSST were stronger in APOE $\varepsilon 4$ non-carriers $\left(\beta=0.081 ; 95 \% \mathrm{Cl}: 0.045,0.117 ; P=1.0 \times 10^{-5}\right)$, whereas carriers performed better in STROOP $(\beta=-0.074 ; 95 \% \mathrm{Cl}:-0.140,-0.009 ; P=0.03)$. Causal associations were found for STROOP only $(\beta=-0.598$ per s.d.-increase of TL; 95\% Cl: $-1.125,-0.072 ; P=0.026)$, with a larger effect in $\varepsilon 4$-carriers $(\beta=-0.699 ; 95 \% \mathrm{Cl}:-1.330,-0.069 ; P=0.03)$. Two-sample replication analyses using CHARGE summary statistics showed causal effects between TL and general cognitive function and DSST, but not with STROOP. In conclusion, we suggest causal effects from longer TL on better cognitive performance, where $A P O E$ \&4-carriers might be at differential risk.

Translational Psychiatry (2017) 7, e1100; doi:10.1038/tp.2017.73; published online 18 April 2017

\section{INTRODUCTION}

Telomeres, short DNA sequences at the end of chromosomes, are considered markers of biological age. Cell replication and oxidative stressors contribute to the loss of telomere nucleotides over time; below critical length, cellular senescence will follow. ${ }^{1}$ An increasing number of studies have shown the importance of telomere length (TL) in ageing, specifically in the development of dementia and cognitive impairment. ${ }^{2-8}$ Using a relatively large group of non-demented older individuals, Yaffe et al. ${ }^{3}$ demonstrated an association between longer telomeres and higher score in the digit symbol substitution test (DSST) - a measure of processing speed-at baseline. After seven years, the individuals with longer telomeres at baseline performed better in the modified mini-mental state exam (MMSE) but not in DSST.

\footnotetext{
${ }^{1}$ Department of Medical Epidemiology and Biostatistics, Karolinska Institutet, Stockholm, Sweden; ${ }^{2}$ Department of Epidemiology, Erasmus University Medical Center, Rotterdam, The Netherlands; ${ }^{3}$ Department of Internal Medicine, Erasmus University Medical Center, Rotterdam, The Netherlands; ${ }^{4}$ Department of Molecular Epidemiology, Leiden University Medical Center, Leiden, The Netherlands; ${ }^{5}$ Max Planck Institute for Biology of Ageing, Cologne, Germany; ${ }^{6}$ Centre for Genomic and Experimental Medicine, Institute of Genetics and Molecular Medicine, University of Edinburgh, Edinburgh, UK; ${ }^{7}$ Centre for Cognitive Ageing and Cognitive Epidemiology, University of Edinburgh, Edinburgh, UK; ${ }^{8}$ Queensland Brain Institute, University of Queensland, Brisbane, QLD, Australia; ${ }^{9} \mathrm{MRC}$ Unit for Lifelong Health and Ageing at UCL, London, UK; ${ }^{10}$ Department of Statistics, Umeå University, Umeå, Sweden; ${ }^{11}$ QIMR Berghofer Medical Research Institute, Brisbane, QLD, Australia; ${ }^{12}$ Gerontology Research Center, Faculty of Sport and Health Sciences, University of Jyväskylä, Jyväskylä, Finland; ${ }^{13}$ Department Biological Psychology, Vrije Universiteit, Amsterdam, The Netherlands; ${ }^{14}$ Department of Gerontology and Geriatrics, Leiden University Medical Center, Leiden, The Netherlands; ${ }^{15}$ Department of Medical Biosciences, Umeå University, Umeå, Sweden; ${ }^{16} \mathrm{NIHR}$ Newcastle Biomedical Research Centre \& Unit, Institute of Neurosciences, Newcastle University, Campus for Ageing and Vitality, Newcastle upon Tyne, UK; ${ }^{17}$ Department of Clinical Sciences, Umeå University, Umeå, Sweden; ${ }^{18}$ Department of Psychology, University California Riverside, Riverside, CA, USA; ${ }^{19}$ Department of Cardiovascular Sciences, University of Leicester, Leicester, UK; ${ }^{20}$ National Institute for Health Research Leicester Cardiovascular Biomedical Research Unit, Glenfield Hospital, Leicester, UK; ${ }^{21}$ Newcastle University Institute for Ageing, Institute for Cell \& Molecular Biosciences, Newcastle upon Tyne, UK; ${ }^{22}$ Centre for Advanced Imaging, University of Queensland, Brisbane, QLD, Australia; ${ }^{23}$ Department of Public Health, Clincum, University of Helsinki, Helsinki, Finland; ${ }^{24}$ Institute for Molecular Medicine Finland (FIMM), University of Helsinki, Helsinki, Finland; ${ }^{25}$ National Institute for Health and Welfare (THL), Helsinki, Finland; ${ }^{26}$ Institute of Health and Biomedical Innovation, Queensland University of Technology, Brisbane, QLD, Australia; ${ }^{27}$ Department of Radiation Sciences, Umeå University, Umeå, Sweden; ${ }^{28}$ Department of Integrative Medical Biology (IMB), Umeå University, Umeå, Sweden; ${ }^{29}$ Umeå center for Functional Brain Imaging, Umeå University, Umeå,

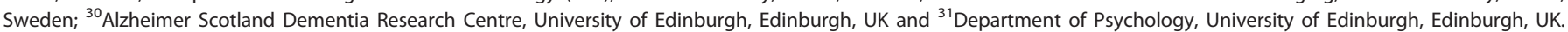
Correspondence: Dr S Hägg, Department of Medical Epidemiology and Biostatistics (MEB), Karolinska Institutet, Box 281, Stockholm 171 77, Sweden.

E-mail: Sara.hagg@ki.se
}

Deceased.

Received 13 February 2017; accepted 13 February 2017 


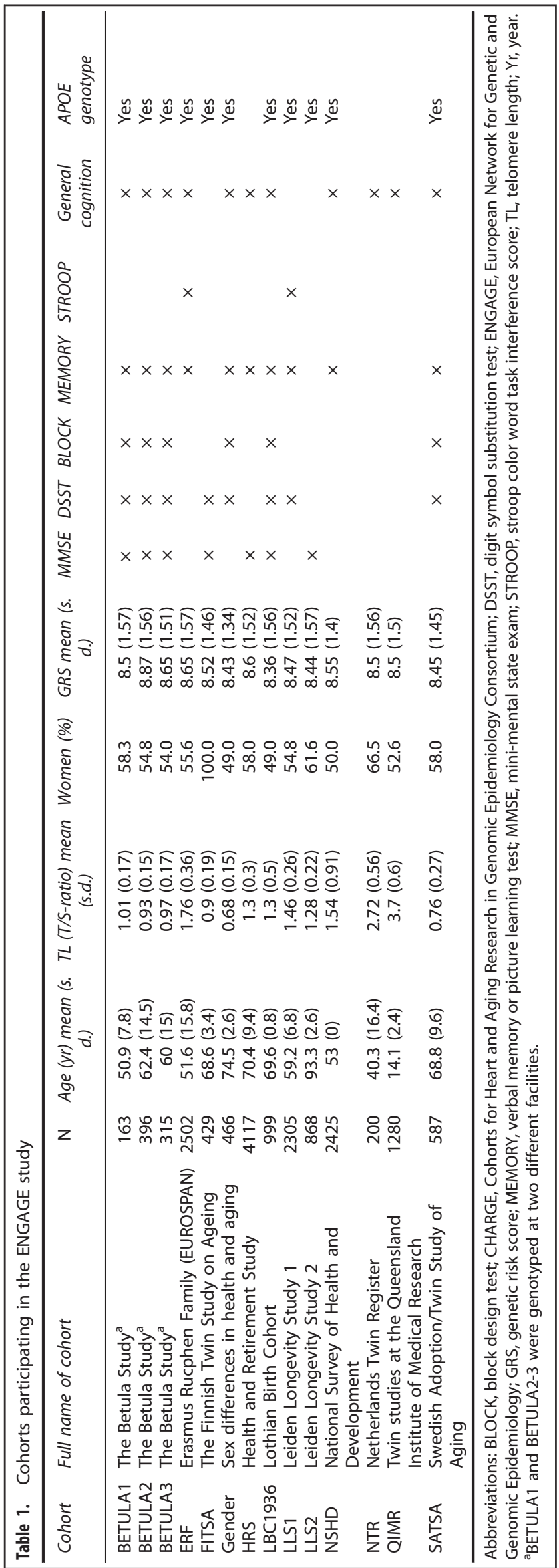

Cohen-Manheim et al. ${ }^{8}$ investigated TL in young adults and found faster attrition rates with poorer mid-life general- and domainspecific cognitive performance but no association with baseline TL. In addition, a cross-sectional study of non-demented individuals concluded that $A P O E$ \&4-carriers had longer TL but faster attrition rates indicating abnormal cell turnover. ${ }^{9}$ Other studies have also shown associations between $\mathrm{TL}$ and cognition with conflicting results or were underpowered. ${ }^{4,6,10,11}$ Hence, the underlying mechanisms by which telomeres may be involved in cognitive performance are complex, and larger efforts are needed to elucidate this relationship. Moreover, it is still unclear whether short telomeres are a cause, consequence or both for cognitive impairment.

One way to predict a causal association is to conduct a Mendelian Randomization (MR) study, ${ }^{12}$ in which genetic markers are used as proxies for an exposure (TL), to investigate an unbiased effect on an outcome (cognitive performance). Because genetic variants are randomly assorted at meiosis, they are generally free from conventional confounding and hence the MR study design is often referred to as nature's own clinical trial. ${ }^{13}$

We hypothesized that $\mathrm{TL}$ is an indicator of cellular stability, which as such affects functioning throughout the body, including performance on all types of cognitive traits. ${ }^{14}$ In addition, individuals carrying the $A P O E \varepsilon 4$ allele are more susceptible to cognitive impairment and are therefore of special interest. ${ }^{15}$ The objective of our study was to conduct a meta-analytic MR study of the association between TL and six cognitive traits in 12 European ancestry cohorts $(N=17052)$. A secondary aim was to stratify on $A P O E \varepsilon 4$ genotype to investigate if carriers were at different risks given their worse cognitive ability. Most cohorts were enrolled through the European Network of Genomic and Genetic Epidemiology (ENGAGE) Consortium. Telomere measurements were performed by $\mathrm{qPCR}$ and a genetic risk score (GRS) with seven genetic variants associated with $\mathrm{TL}^{16}$ was calculated. Observational- as well as causal estimates were subsequently obtained using an MR design. ${ }^{17}$ In a replication effort, summary statistics from the Cohorts for Heart and Aging Research in Genomic Epidemiology (CHARGE) Consortium for the genetic associations with three cognitive traits ${ }^{18,19}$ were included in a twosample MR approach. ${ }^{20}$

\section{MATERIALS AND METHODS}

Study samples

Twelve cohorts with a total of 17052 individuals (Table 1), all with European ancestry populations, participated in the ENGAGE effort. The sample-size weighted mean of age was 59.2 years with s.d. $=8.8$. Most cohorts contributed data measured at mid-life or older. The Leiden Longevity Study 2 (LLS2) was the oldest cohort (mean age $=93.3$ years). The Netherlands Twin Register (NTR) included middle-aged adults (mean age $=40.3$ years, s.d. $=16.4$ ) and QIMR (Twin studies at the Queensland Institute of Medical Research) included adolescents only (mean age $=14.1$ years, s.d. $=2.4$ ). All but one study showed a fairly even proportion of sexes (range: 49-67\% women); FITSA (The Finnish Twin Study on Ageing) included women only. Additional study-specific details are found in Supplementary Table 1.

\section{Cognitive traits}

Six different cognitive traits were tested in a combined meta-analysis of the ENGAGE cohorts: (1) general cognitive function; (2) MMSE; (3) processing speed with DSST or the variant symbol digit substitution task; (4) visuospatial functioning with block design test (BLOCK); (5) episodic memory by either verbal learning or picture learning tests (MEMORY); and (6) executive functioning using Stroop interference score (STROOP). Detailed descriptions of the different cognitive traits are found in the supplement (Supplementary Table 3). All cohorts participated with at least one cognitive trait; no single cohort had all of them (Table 1). 
Telomere length measurements

Telomere length was measured in leukocytes in whole blood/buffy coat except for the HRS study, which used measurements from saliva. DNA from saliva derives for the most part ( 74\%) from leukocytes, ${ }^{21}$ and TL measurements from blood and saliva have been reported to have good correlations $(R=0.72){ }^{22}$ Standard $\mathrm{qPCR}$ techniques for TL measurement were applied as described by Cawthon ${ }^{23}$ with minor modifications in the Lothian Birth Cohort (LBC) ${ }^{11}$ and BETULA. ${ }^{24}$ In brief, telomere (T) and single copy gene (S) quantity were measured and a T/S-ratio was calculated. One or several reference samples were included in all runs and a relative telomere length was calculated for each sample.

\section{Genotyping}

Information on genotyping platform, quality control and single-nucleotide polymorphisms (SNPs) used in each cohort is available in the supplement (Supplementary Data and Supplementary Table 2). An additive unweighted GRS was calculated for each individual by summarizing the number of risk alleles from seven different loci (TERC, TERT, NAF1, OBFC1, ZNF208, RTEL1 and ACYP2) where SNPs (rs10936599, rs2736100, rs7675998, rs9420907, rs8105767, rs755017 and rs11125529) have been found to associate with TL. ${ }^{16}$ Investigations of possible pleiotropic effects from the different genetic variants used in the GRS are discussed in the supplement (Supplementary Data). Four cohorts (ERF (Erasmus Rucphen Family), LLS, NTR and QIMR) from the current effort contributed to the original genomewide association study (GWAS) of TL (see Supplementary Data for further discussions on implications to our study). No associations between any of the seven TL genes and cognitive traits have been tested thus far as judged from the GWAS Catalog. ${ }^{25}$ Genetic variants were prioritized as (1) directly genotyped, (2) imputed with good quality, (3) proxies with $r^{2}>0.8$ and (4) imputed from summary statistics. The weighted mean of the GRS was 8.55 with s.d. $=1.51$ (Table 1). APOE genotype was assessed separately and available in most cohorts (Table 1).

\section{Replication data}

Summarized results from the CHARGE Consortium's meta-analyses of genome-wide association studies between genotypes and general cognitive function $(N=53949){ }_{1}^{18}$ processing speed by a meta-analysis of four tests of processing speed including the DSST $(N=32088)^{19}$ and executive functioning (STROOP; $N=7726)^{19}$ were used to assess causal associations from the same seven TL associated genetic variants. ${ }^{16}$ The CHARGE cohorts were all of European ancestry and participants were aged 45 years or older. Some ENGAGE cohorts contributed to CHARGE analyses of general cognitive function: BETULA1, ERF, HRS, and LBC1936; DSST and STROOP: ERF; and DSST: LBC1936 (Table 1; see Supplementary Data for further discussions on implications to our study). The general cognitive function phenotype was created as a composite score of multiple cognitive tests ${ }^{18}$ from principal component analysis. The processing speed variable was created from DSST and three similar tests, and executive functioning was assessed by either Trail Making tests or Stroop color and word interference tests. ${ }^{19}$ Effect sizes were missing for DSST and STROOP; hence results were presented in Z-scores only.

\section{Statistical analyses}

All variables (TL and cognitive traits) were $Z$-transformed with subtraction of the mean and division of s.d. to enable comparisons across cohorts. Age groups, instead of continuous age, were defined and used as covariates to allow for non-linear age effects: (1) 0-29 years; (2) 30-59 years; (3) 60-79 years; and (4) $80+$ years. Cohorts with information on APOE genotype performed additional analyses stratified on $\varepsilon 4$-carriers $(\varepsilon 4 / \varepsilon 4$ and $\varepsilon 4 / \varepsilon 3)$ and non-carriers $(\varepsilon 3 / \varepsilon 3, \varepsilon 2 / \varepsilon 3$ and $\varepsilon 2 / \varepsilon 2)$. Individuals with the genotype $\varepsilon 2 /$ $\varepsilon 4$ are excluded from the analysis. All models are described in detail in the Supplementary Data; briefly, all cohorts contributed with summary data from three different models as depicted in Figure 1. Linear regressions were fitted for the associations of (1) TL on cognitive trait (TL-trait), (2) GRS on TL (GRS-TL), and (3) GRS on cognitive trait (GRS-trait). All models were adjusted for sex, age group and study-specific covariates. Effect estimates from all models were pooled across cohorts via fixed-effect meta-analysis, except when evidence was found for statistically significant heterogeneity $(P$-value $<0.05)$, in which case a random-effects meta-analysis was performed instead. Then, instrumental variable (IV) analysis was conducted by calculating a Wald-type causal estimate for the effect of TL on each cognitive trait (IV - trait=GRS - trait/GRS-TL). Effect differences between

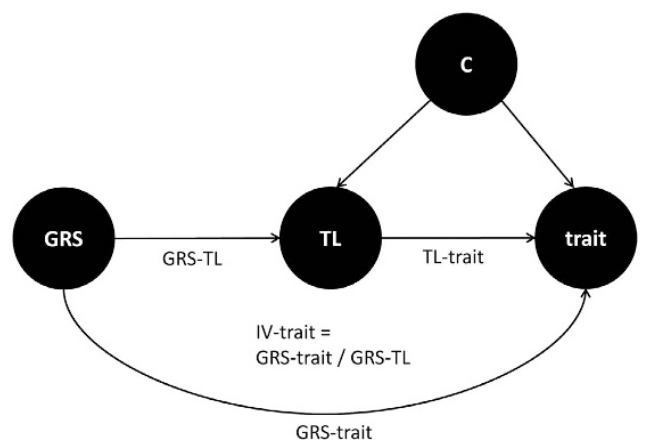

Figure 1. Graph describing the design of the study. A genetic risk score (GRS) for telomere length (TL) is used in the instrumental variable (IV) analysis to determine the predicted effect of TL on different cognitive traits (IV-trait). The Mendelian Randomization design allows for calculation of an estimate independent of confounders (C) in comparison to the observed effect estimated between TL and cognitive traits (TL-trait).

observed (TL-trait) and causal/predicted (IV-trait) estimates were calculated by subtracting the causal beta from the observational beta in a Z-test (Supplementary Data). Stratified analyses on APOE genotype were done similarly. For summary statistics data from CHARGE, a causal estimate was calculated as described by Burgess et al. ${ }^{20}$ (Supplementary Data). Crude $P$-values are presented for all associations, that is, no multiple testing corrections have been applied.

\section{RESULTS}

Observational analyses of telomere length and cognitive traits Significant associations, supporting the relationship between longer telomeres and better cognitive ability, were seen between TL and MMSE, DSST and STROOP using fixed-effects meta-analysis (Table 2, Figure 2). Positive associations were observed for MMSE (0.025 per s.d.-increase in TL; 95\% confidence interval (Cl) 0.002, $0.047)$ and DSST $(0.051 ; 95 \% \mathrm{Cl} 0.024,0.077)$. For STROOP, a negative beta $(-0.053$ per s.d.-increase in $\mathrm{TL} ; 95 \% \mathrm{Cl}-0.087$, - 0.018) was seen, which was in accordance with the hypothesis that longer telomeres are associated with shorter time for completion of the Stroop interference test. However, after corrections for multiple comparisons, the association with MMSE was not significant.

\section{Genetic risk score for telomere length}

The combined effect of the GRS on TL was -0.048 s.d. - change of TL per allele $\left(95 \% \mathrm{Cl}\right.$ : $-0.064,-0.032, \quad P$-value $\left.=4.0^{*} 10^{-9}\right)$ calculated using random-effects meta-analysis (Supplementary Data: Supplementary Figure S1). The corresponding F-statistic was 36 , indicating that the GRS-TL estimate provided a sufficiently strong instrument for further use in IV analyses. ${ }^{26}$ Although heterogeneity was detected, all cohorts showed negative effect sizes ranging from -0.01 to -0.13 (Supplementary Table S1). Additional tests investigating possible pleiotropic effects for SNPtrait associations were done and found no evidence of such (Supplementary Data).

Instrumental variable analyses of telomere length and cognitive traits

Instrumental variable analyses for causal associations of TL on cognitive performance were conducted for all cognitive traits. Only the association between TL and STROOP was found to be causal (Table 2), and for each s.d.-decrease in TL an effect change of -0.60 in Stroop score was detected $(95 \% \mathrm{Cl}$ : $-1.12,-0.07$, $P$-value $=0.026)$. However, the association would not be significant 
Table 2. Associations between predicted and observed telomere length and different cognitive traits

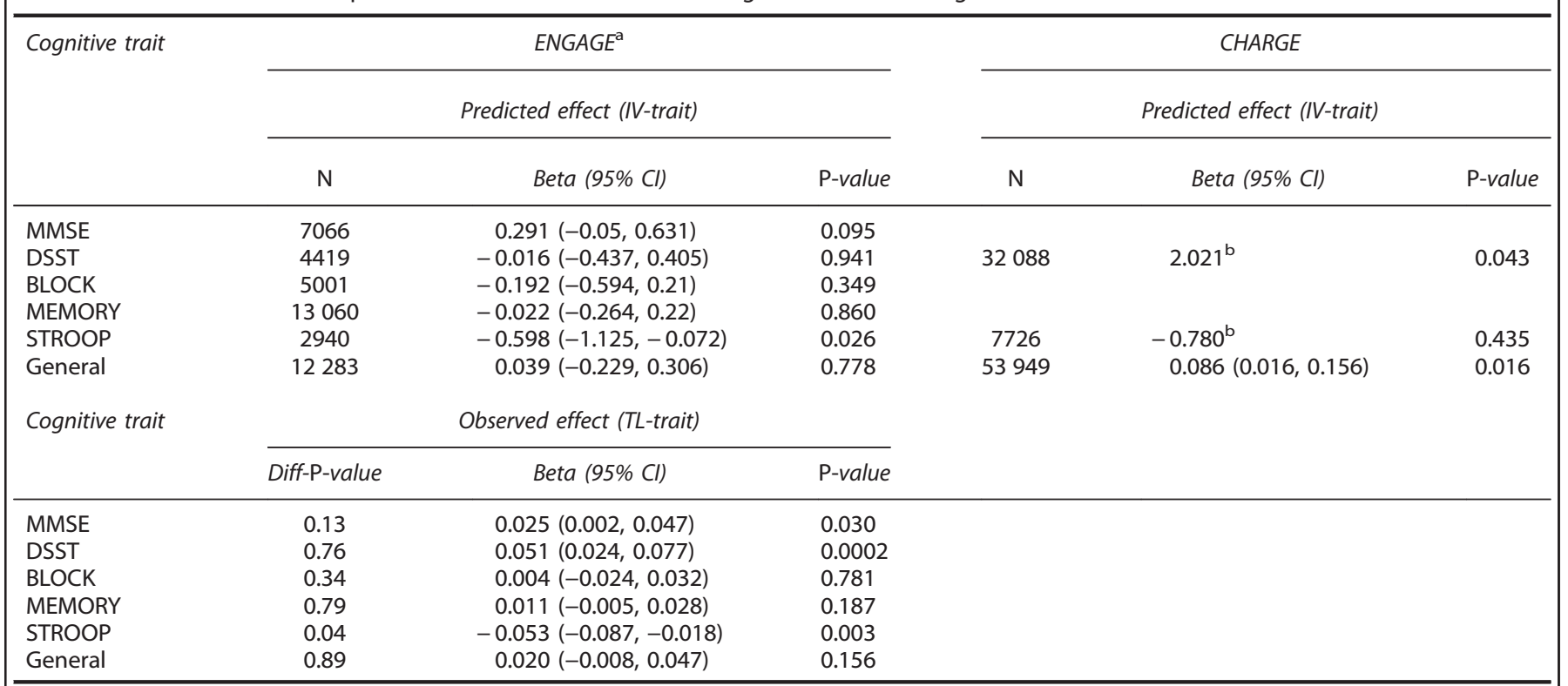

Abbreviations: BLOCK, Block-design test; $\mathrm{Cl}$, confidence interval; Diff- $P$-value, tests for difference in estimators between observed and predicted effects; DSST, Digit-symbol substitution test; General, General cognitive performance; IV, instrumental variable; MEMORY, Verbal memory or Picture learning test; MMSE,

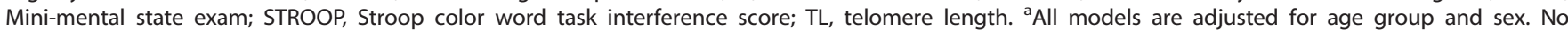
adjustments for multiple testing have been done on the reported $P$-values. ${ }^{b} Z$-scores, effect sizes are missing.

a

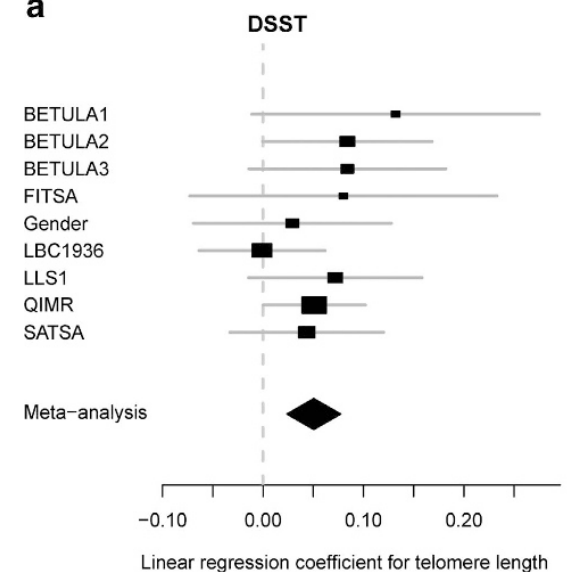

b

BETULA1
BETULA2
BETULA3
FITSA
Gender
HRS
LBC1936
LLS2
SATSA

Meta-analysis

C

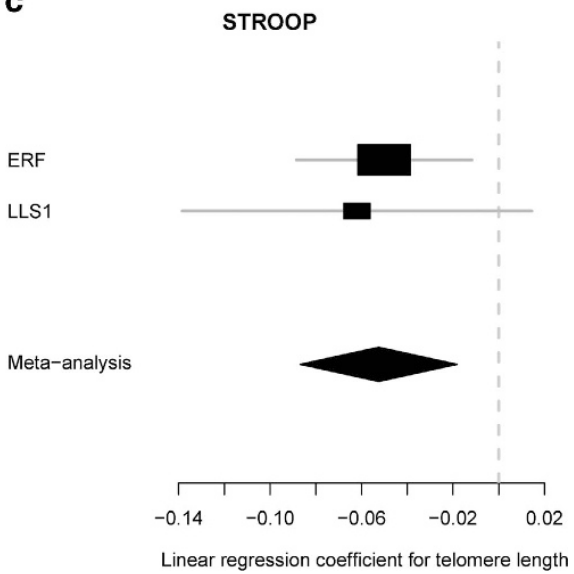

Figure 2. Observed effects between telomere length and cognitive traits. Fixed-effects meta-analyses were performed across cohorts and domains for all cognitive traits. Significant effects (s.d.-change in cognitive score for an s.d.-change in telomere length (TL)) were found for (a). digit symbol substitution test tapping processing speed (DSST), (b). Mini-mental state exam (MMSE), and (c). Stroop interference score tapping executive functioning (STROOP). All models were adjusted for age group, sex and study-specific covariates.

after multiple testing adjustments. The difference in effect sizes between observational and causal betas for STROOP was statistically significant (Table 2).

\section{Stratified analyses}

Stratified meta-analyses were performed for APOE $\varepsilon 4$-carriers $(n \leqslant 2380)$ and $\varepsilon 4$ non-carriers $(n \leqslant 5669)$ separately (Supplementary Data). Observational associations were seen between TL and DSST in non-carriers $\left(\beta=0.081,95 \% \mathrm{Cl}: 0.045,0.117, P\right.$-value $\left.=1 \times 10^{-5}\right)$ and with better performance for STROOP in carriers $(\beta=-0.074$, 95\% Cl: $-0.140,-0.009, P$-value $=0.027$ ) (Supplementary Table S4). A causal association between long telomeres and better performance for STROOP was detected amongst APOE ع4-carriers $(\beta=-0.70,95 \% \mathrm{Cl}:-1.33,-0.07, P$-value $=0.030)$, although the finding would not hold after multiple testing adjustments. No other causal effects for TL on cognitive traits were seen in either carriers or non-carriers (Supplementary Table S6).

Replication analyses

In replication efforts, two-sample MR analyses were carried out using summary statistics from CHARGE GWAS on general cognitive function, DSST and STROOP. Data from the TL GWAS were used for the genetic instrument (Supplementary Data). Results provided evidence for a causal association between longer leukocyte TL and better general cognitive function $(\beta=0.086$ per s.d.-increase of $\mathrm{TL}$, 95\% Cl: 0.016-0.156, $P$-value $=0.016$, Table 2 ) and better DSST 
scoring $(Z$-score $=2.02, P$-value $=0.043$, Table 2$)$. No evidence for a causal effect by TL on STROOP was found (Table 2).

\section{DISCUSSION}

In the present study, we provide evidence for observational and causal associations between longer telomeres and better cognitive performance. By conducting a large meta-analysis of 12 cohorts from European ancestry populations with measured telomeres and assessments of cognitive function, we were able to observe associations between TL and better scoring on MMSE, DSST and STROOP. Moreover, APOE \&4-carriers seemed to have different effects for the observed association with worse performance in DSST but better in STROOP. The association between longer telomeres and faster completion of the Stroop interference test was also found to be significant in causal analysis for all individuals and in APOE $\varepsilon 4$-carriers only. However, none of the significant causal associations detected for STROOP passed multiple testing corrections. Hence, in line with this, using summary data from CHARGE, we found support for a causal association from TL on general cognitive function and DSST, but not on STROOP.

In the biology of aging, telomere length has long been considered as a biomarker reflecting the underlying cellular state. Recently, however, several research papers have presented evidence of telomeres being involved in the process of cellular senescence causing increased risk of disease. ${ }^{5,16,27}$ Moreover, other studies suggest telomeres might even elongate in somatic cells to maintain cellular stability, ${ }^{21,28,29}$ although this phenomenon could be partly explained by leukocyte turnover or imprecise measurements. Nevertheless, telomere biology has implications for the aging processes and studies are warranted to elucidate the full complexity.

With this effort we demonstrate several observational associations between $\mathrm{TL}$ and cognitive traits, both confirming earlier studies and presenting new links. General cognitive function is usually operationalized as a composite score across a number of diverse cognitive domains capturing most of the cognitive variation. ${ }^{18}$ As an overall measure of cognition it also predicts mortality ${ }_{i}^{30}$ likewise, the length of telomeres can be used to predict mortality. ${ }^{31,32}$ Thus, if both general cognitive function and TL serve as valid biomarkers of aging, associations between these markers are expected, although causality needs to be further investigated. In the ENGAGE data $(N=12283)$, we were not able to detect any association between general cognitive function and $T L$, but using the CHARGE summary data $(N=53949)$ we provided evidence for a causal association. It is likely that the ENGAGE analysis was low in power; effect sizes had overlapping Cl's. A possible biological mechanism for a causal association could be explained by overall body frailty; the lengths of telomeres are important for maintaining cellular stability at old age and hence also important for biological aging processes such as decline in cognitive performance. ${ }^{1}$ Causal links have also been demonstrated using animal models. A mouse with telomerase deficiency, expressing accelerated aging with malfunctioning tissue repair and impaired neurological function, had restored functions again upon telomerase reactivation. ${ }^{33}$

The DSST test assesses processing speed required to translate a code of symbols and digits as fast as possible in a given time frame. Processing speed has been demonstrated to have a steady, almost linear decline with advancing age, and its decline leads other forms of cognitive decline. ${ }^{34,35}$ Hence, in light of this it is not surprising that we, and others, ${ }^{3}$ detect a fairly stable observational association of longer telomeres and better DSST scoring. The MR analysis did not indicate a causal association in our samples $(N=4419)$; on the other hand, when increasing power using CHARGE data $(N=32088)$ we were able to find support for a positive causal relationship from longer TL on DSST scoring.
Moreover, $A P O E \varepsilon 4$ non-carriers scored better on the test with a larger effect size seen in observational analysis from TL on DSST. Thus, as $A P O E$ genotype is important for elucidating different risk groups for many age-related phenotypes, it is possible that it applies to TL dynamics and cognitive performance as well.

Yaffe et al. ${ }^{3}$ showed that longer TL at baseline gave less longitudinal decline in MMSE, and we presented cross-sectional evidence from observational associations in line with these findings; longer TL is consistent with better MMSE scoring. However, while causal estimates support these associations, the $\mathrm{Cl}^{\prime}$ 's were wide and results did not reach statistical significance. Unfortunately, CHARGE data on MMSE were not available for replication analysis.

The STROOP variable taps the executive functioning by a combined color and word test to be completed as quickly as possible. To the best of our knowledge, there has been only one earlier small study investigating baseline and attrition TL associations with executive functioning, with inconclusive results. ${ }^{8}$ Our ENGAGE analysis included 2940 individuals where we found both an observational and causal association between longer telomeres and faster completion of the Stroop test. The large effect size difference was however disturbing and not explained by additional adjustments for smoking and alcohol (Supplementary Data). Further, causal associations did not hold after multiple testing corrections and when using the two-sample approach including the larger CHARGE data $(N=7726)$ we could not replicate the association, although the effects were in the same direction. Hence, it is possible that the STROOP finding observed in the ENGAGE data is a false discovery. In addition, the stratified analyses by $A P O E \& 4$ genotype found $\varepsilon 4$-carriers to perform better, which is contradictory to what would be expected.

The strength of this study is the effort of combining multiple European cohorts with $\mathrm{TL}$, cognitive and genetic data available as well as $A P O E$ genotype. By doing so, we were able to detect patterns of associations for different cognitive traits that would not be possible to find in single study analyses. Moreover, we included large-scale CHARGE GWAS data sets to perform twosample MR analyses as replication. The weaknesses of the study include generalizability, as the analyses were performed solely in European ancestry populations, and some of the cohorts were included in both ENGAGE and CHARGE analyses as described in the supplement. Moreover, heterogeneity due to different tissues used (blood and saliva) and lab-specific technical variances (TL estimates from all 12 cohorts were done in five different labs) may have driven the results toward null. Another limitation relates to the three assumptions for conducting MR studies, which have been considered as follows: (1) a strong genetic instrument should be demonstrated between the GRS and TL (GRS-TL) which we have (F-statistic = 36); (2) the genetic instrument should not be confounded by e.g., age and sex (unlikely considering the randomization of alleles at conception); and (3) pleiotropic effects (when other pathways exist from the TL SNPs to the outcome (cognitive trait) without going through the intermediate phenotype (TL)) from the SNPs included in the GRS should be ruled out as much as possible. We did not find evidence for pleiotropic effects (Supplementary Data). Finally, also worth mentioning are the relatively weak $P$-values for some of the associations. The observational association for MMSE would not hold after Bonferroni correction of the $P$-value for the six cognitive traits tested, likewise for the causal associations found for STROOP.

To conclude, this study demonstrates an overall picture of the importance of biological aging processes such as TL dynamics for maintaining cognitive function throughout life. More specifically, we were able to show observational as well as causal associations between $\mathrm{TL}$ and different cognitive traits that have never been elucidated before. Hence, the current effort presents new important pieces of evidence for the continued search for a 
better understanding of the biology behind aging and the factors explaining healthy aging.

\section{CONFLICT OF INTEREST}

The authors declare no conflict of interest.

\section{ACKNOWLEDGMENTS}

European Network for Genetic and Genomic Epidemiology (ENGAGE) with FP7HEALTH-F4-2007 grant agreement nr. 201413. The Cohorts for Heart and Aging Research in Genomic Epidemiology Consortium (CHARGE) which is supported in part by the National Heart, Lung and Blood Institute grant HL105756 and the neuroCHARGE cognitive working group through the National Institute on Aging grant AG033193. All cohort-specific acknowledgments are found in the Supplementary Data.

\section{REFERENCES}

1 Blackburn EH, Epel ES, Lin J. Human telomere biology: a contributory and interactive factor in aging, disease risks, and protection. Science 2015; 350: 1193-1198.

2 Wikgren M, Karlsson T, Lind J, Nilbrink T, Hultdin J, Sleegers K et al. Longer leukocyte telomere length is associated with smaller hippocampal volume among non-demented APOE epsilon3/epsilon3 subjects. PLoS ONE 2012; 7: e34292.

3 Yaffe K, Lindquist K, Kluse M, Cawthon R, Harris T, Hsueh WC et al. Telomere length and cognitive function in community-dwelling elders: findings from the Health ABC Study. Neurobiol Aging 2011; 32: 2055-2060.

4 Zekry D, Herrmann FR, Irminger-Finger I, Graf C, Genet C, Vitale AM et al. Telomere length and ApoE polymorphism in mild cognitive impairment, degenerative and vascular dementia. J Neurol Sci 2010; 299: 108-111.

5 Zhan Y, Song C, Karlsson R, Tillander A, Reynolds CA, Pedersen NL et al. Telomere length shortening and Alzheimer disease-a Mendelian randomization study. JAMA Neurology 2015; 72: 1202-1203.

6 Devore EE, Prescott J, De Vivo I, Grodstein F. Relative telomere length and cognitive decline in the Nurses' Health Study. Neurosci Lett 2011; 492: 15-18.

7 Honig LS, Kang MS, Schupf N, Lee JH, Mayeux R. Association of shorter leukocyte telomere repeat length with dementia and mortality. Arch Neurol 2012; 69: 1332-1339.

8 Cohen-Manheim I, Doniger GM, Sinnreich R, Simon ES, Pinchas R, Aviv A et al. Increased attrition of leukocyte telomere length in young adults is associated with poorer cognitive function in midlife. Eur J Epidemiol 2016; 31: 147-157.

9 Wikgren M, Karlsson T, Nilbrink T, Nordfjall K, Hultdin J, Sleegers K et al. APOE epsilon4 is associated with longer telomeres, and longer telomeres among epsilon4 carriers predicts worse episodic memory. Neurobiol Aging 2012; 33: 335-344.

10 Zekry D, Herrmann FR, Irminger-Finger I, Ortolan L, Genet C, Vitale AM et al. Telomere length is not predictive of dementia or $\mathrm{MCl}$ conversion in the oldest old. Neurobiol Aging 2010; 31: 719-720.

11 Harris SE, Marioni RE, Martin-Ruiz C, Pattie A, Gow AJ, Cox SR et al. Longitudinal telomere length shortening and cognitive and physical decline in later life: The Lothian Birth Cohorts 1936 and 1921. Mech Ageing Dev 2016; 154: 43-48.

12 Palmer TM, Sterne JA, Harbord RM, Lawlor DA, Sheehan NA, Meng S et al. Instrumental variable estimation of causal risk ratios and causal odds ratios in Mendelian randomization analyses. Am J Epidemiol 2011; 173: 1392-1403.

13 Burgess S, Butterworth A, Malarstig A, Thompson SG. Use of Mendelian randomisation to assess potential benefit of clinical intervention. BMJ 2012; 345: e7325.

14 Clouston SA, Brewster P, Kuh D, Richards M, Cooper R, Hardy R et al. The dynamic relationship between physical function and cognition in longitudinal aging cohorts. Epidemiol Rev 2013; 35: 33-50.

15 Salmon DP, Ferris SH, Thomas RG, Sano M, Cummings JL, Sperling RA et al. Age and apolipoprotein $\mathrm{E}$ genotype influence rate of cognitive decline in nondemented elderly. Neuropsychology 2013; 27: 391-401.

16 Codd V, Nelson CP, Albrecht E, Mangino M, Deelen J, Buxton JL et al. Identification of seven loci affecting mean telomere length and their association with disease. Nat Genet 2013; 45: 422-427.
17 Hagg S, Fall T, Ploner A, Magi R, Fischer K, Draisma HH et al. Adiposity as a cause of cardiovascular disease: a Mendelian randomization study. Int J Epidemiol 2015; 44: $578-586$.

18 Davies G, Armstrong N, Bis JC, Bressler J, Chouraki V, Giddaluru S et al. Genetic contributions to variation in general cognitive function: a meta-analysis of genome-wide association studies in the CHARGE consortium $(\mathrm{N}=53949)$. Mol Psychiatry 2015; 20: 183-192.

19 Ibrahim-Verbaas CA, Bressler J, Debette S, Schuur M, Smith AV, Bis JC et al. GWAS for executive function and processing speed suggests involvement of the CADM2 gene. Mol Psychiatry 2016; 21: 189-197.

20 Burgess S, Scott RA, Timpson NJ, Davey Smith G, Thompson SG. Consortium E-I Using published data in Mendelian randomization: a blueprint for efficient identification of causal risk factors. Eur J Epidemiol 2015; 30: 543-552.

21 Cai N, Chang S, Li Y, Li Q, Hu J, Liang J et al. Molecular signatures of major depression. Curr Biol 2015; 25: 1146-1156.

22 Mitchell C, Hobcraft J, McLanahan SS, Siegel SR, Berg A, Brooks-Gunn J et al. Social disadvantage, genetic sensitivity, and children's telomere length. Proc Natl Acad Sci USA 2014; 111: 5944-5949.

23 Cawthon RM. Telomere measurement by quantitative PCR. Nucleic Acids Res 2002; 30: e47.

24 Degerman S, Domellof M, Landfors M, Linder J, Lundin M, Haraldsson S et al. Long leukocyte telomere length at diagnosis is a risk factor for dementia progression in idiopathic parkinsonism. PLOS ONE 2014; 9: e113387.

25 Welter D, MacArthur J, Morales J, Burdett T, Hall P, Junkins $\mathrm{H}$ et al. The NHGRI GWAS Catalog, a curated resource of SNP-trait associations. Nucleic Acids Res 2014; 42(Database issue): D1001-D1006.

26 Burgess S, Thompson SG. Use of allele scores as instrumental variables for Mendelian randomization. Int J Epidemiol 2013; 42: 1134-1144.

27 Zhang C, Doherty JA, Burgess S, Hung RJ, Lindstrom S, Kraft P et al. Genetic determinants of telomere length and risk of common cancers: a Mendelian randomization study. Hum Mol Genet 2015; 24: 5356-5366.

28 Kato S, Shiels PG, McGuinness D, Lindholm B, Stenvinkel P, Nordfors L et al. Telomere attrition and elongation after chronic dialysis initiation in patients with end-stage renal disease. Blood Purif 2016; 41: 25-33.

29 Aviv A, Chen W, Gardner JP, Kimura M, Brimacombe M, Cao X et al. Leukocyte telomere dynamics: longitudinal findings among young adults in the Bogalusa Heart Study. Am J Epidemiol 2009; 169: 323-329.

30 Calvin CM, Deary IJ, Fenton C, Roberts BA, Der G, Leckenby N et al. Intelligence in youth and all-cause-mortality: systematic review with meta-analysis. Int J Epidemiol 2011; 40: 626-644.

31 Deelen J, Beekman M, Codd V, Trompet S, Broer L, Hagg S et al. Leukocyte telomere length associates with prospective mortality independent of immunerelated parameters and known genetic markers. Int J Epidemiol 2014; 43: 878-886.

32 Bakaysa SL, Mucci LA, Slagboom PE, Boomsma DI, McClearn GE, Johansson B et al. Telomere length predicts survival independent of genetic influences. Aging Cell 2007; 6: 769-774.

33 Jaskelioff $\mathrm{M}$, Muller FL, Paik JH, Thomas E, Jiang S, Adams AC et al. Telomerase reactivation reverses tissue degeneration in aged telomerase-deficient mice. Nature 2011; 469: 102-106.

34 Finkel D, Reynolds CA, McArdle JJ, Pedersen NL. Age changes in processing speed as a leading indicator of cognitive aging. Psychol Aging 2007; 22: 558-568.

35 Hagenaars SP, Harris SE, Davies G, Hill WD, Liewald DC, Ritchie SJ et al. Shared genetic aetiology between cognitive functions and physical and mental health in UK Biobank ( $\mathrm{N}=112$ 151) and 24 GWAS consortia. Mol Psychiatry 2016; 21: 1624-1632.

This work is licensed under a Creative Commons Attribution 4.0 International License. The images or other third party material in this
article are included in the article's Creative Commons license, unless indicated otherwise in the credit line; if the material is not included under the Creative Commons license, users will need to obtain permission from the license holder to reproduce the material. To view a copy of this license, visit http://creativecommons.org/licenses/ by/4.0/

(c) The Author(s) 2017

Supplementary Information accompanies the paper on the Translational Psychiatry website (http://www.nature.com/tp) 